\title{
Interactive System of Work Support in Consideration of Worker Competency*
}

\author{
Toshitake TATENO**
}

\begin{abstract}
This paper deals with a work support system that helps novice workers to execute and learn tasks efficiently. In order to realize this system, the interaction process between workers and the support system is discussed. Radio frequency identification (RFID) system is applied for setting flexible interaction processes in consideration of worker competency. Also, a software simulator that measures the capacity of human memory and learning is used to formulate a guideline for the interaction design. Finally, an experiment on the work support system in actual assembly work is conducted. As a result, all subjects were able to complete their tasks without error, even when they attempted the work for the first time, and to learn a series of tasks by the practical operations using the support system. This result confirms the effectiveness of the work support system.
\end{abstract}

Key Words: Operation Planning, Assembly, Computer Integrated Manufacturing (CIM), Work Support, Radio Frequency Identification (RFID), Human Computer Interaction (HCI), Learning Curve

\section{Introduction}

In equipment maintenance, workers deal with many kinds of tasks and sometimes have to troubleshoot with unfamiliar tasks using manuals. However, the number of expert workers is reduced and the number of part time workers is increased. Therefore, work support systems are important in order for workers to execute tasks safely and efficiently.

The author proposes a new work support system that changes the interaction processes considering the worker competency. Table 1 classifies the effects of the system. By using the interactive process actively, the system enables novice workers to execute new tasks and to gain experience in practical work. This leads to not only the prevention of human error and the reduction of work time, but also to learning. Providing much information is not always effective. Timely and suitable instruction is effective for learning because workers need not select the required information from the given information. Novice workers usually require much information to execute tasks and checks to prevent errors. On the other hand, expert workers need little information or checks. In this paper, a

* Received 1st June, 2005 (No. 05-4065)

** Department of System Design, Tokyo Metropolitan University, 1-1 Minami-Ohsawa, Hachioji, Tokyo 192-0397, Japan. E-mail: tateno-toshitake@c.metro-u.ac.jp flexible setting scheme of the instruction processes is proposed to provide work support that is adaptive to a wide range of worker competency.

The interaction processes consist of an instruction process and a checking process. A typical example of instruction is a work manual, and a typical example of checking is a checklist. The required system is an electrical instruction and checking system that adapts to the worker competency. Especially, checking process is important to prevent task omission and misunderstanding. The checking processes should be considered to suit the worker competency.

In this paper, related studies are surveyed in the next section. In section 3, the interactive work support system, which can set checking processes flexibly using RFID, is introduced. In section 4 , in order to design the checking process for novice workers, basic human properties in the work situation are measured using a work simulator, which is like a computer game. Then, in section 5, the

Table 1 Effects of the interactive work support system

\begin{tabular}{|l|l|}
\hline \multirow{3}{*}{$\begin{array}{l}\text { Safety and } \\
\text { Efficient work }\end{array}$} & $\begin{array}{l}\text { Prevention of human error } \\
\text { (Reduction of judgments made by workers) }\end{array}$ \\
\cline { 2 - 2 } Work learning & $\begin{array}{l}\text { Synchronization with production management system } \\
\text { (Reduction of data input tasks) }\end{array}$ \\
\hline \multirow{2}{*}{$\begin{array}{l}\text { Increase in practical experience } \\
\text { (Reduction of initial learning term) }\end{array}$} \\
\cline { 2 - 2 } & $\begin{array}{l}\text { Increase in knowledge of work and products } \\
\text { (Timely and suitable instruction) }\end{array}$ \\
\hline
\end{tabular}


work efficiency and learning effect are confirmed in actual assembly work using a prototype system.

\section{Related Studies}

In order to improve work efficiency and to prevent human error, many kinds of fail-safe mechanisms have been devised in equipments and operation methods. Recently, some work support systems have been introduced to direct the task order to workers and to check the human work by means of a computer system. One such system is the manufacturing execution system (MES) ${ }^{(1)}$. It aims at informative connection of human manual work and automated systems. MES presents the task order that the worker should execute, and the worker returns a signal after finishing the task. However, the present MES has been applied only to the tasks already familiar to workers. It does not accommodate work instruction for a worker to learn new tasks.

There has been some studies on constructing work support systems that enable novice workers to complete new tasks. We have proposed a work support system ${ }^{(2)}$ that presents work instruction based on human motion recognition. Raghavan et al. ${ }^{(3)}$ introduced a system that gives instructions to a worker through an augmented reality system for workers to understand the task intuitively. These systems commonly include interaction between the human and the machine, where a computer presents task information, a worker executes the task, and a computer evaluates it by motion or image recognition. However, the interaction processes of these support systems depend on the tasks that can be clearly recognized by machines. A new support system where interaction processes can be set flexibly is required.

The method of learning by which a novice worker learns tasks by performing practical operations with expert workers is called as on-the-job training (OJT). Typically, in OJT, a student and a trainer work together. The trainer checks the student's work and gives instructions. There are two merits of OJT. One is that OJT enables the avoidance of non-profitable training. Another merit is that trainers give suitable instructions at appropriate times according to the student's skill level. Therefore, the required system is a computer system that replaces the human trainer. There are rare reports dealing with OJT computer systems.

With regard to the training tool by computers, there are many studies in computer-aided instruction (CAI) field. CAI has been developed using artificial intelligence (AI) technology $y^{(4)}$. Many studies to built courseware have been discussed. Besides the intelligent CAI system, simulation-based training has also been developed under the concept of learning by doing ${ }^{(5)}$. A training system for plant operation ${ }^{(6)}$ is practically used with a virtual reality system that provides workers with quasi-experience. However, most CAI systems are used in a training stage, which is a different stage from practical work.

\section{Interactive Work Support System}

\subsection{Concept of the system and realization with RFID}

There are two approaches to checking human tasks by means of a computer system. One is to recognize human motion or assembly states automatically. However, there is some miss-recognition because the worker motion is varies according to the person. Another is to require check action of workers. This approach is adopted for this system to confirm a worker understanding.

Figure 1 shows an example of this approach. When a worker puts a peg into a hole, the system should confirm two things: which peg and which hole are used. Therefore, the system requires the worker to touch a certain point to identify the work piece and position. Then, if the work piece and position are confirmed, the work instruction is indicated. With this function, workers can execute tasks even though they have no work knowledge about the work piece. This type of interaction increases work reliability, but too much confirmation makes the work time longer ${ }^{(7)}$. The instruction and confirmation processes should be reduced by timely and suitable instruction. In this paper, the flexible setting of interaction processes, particularly checking processes, is discussed. A flexible checking process is realized with RFID.

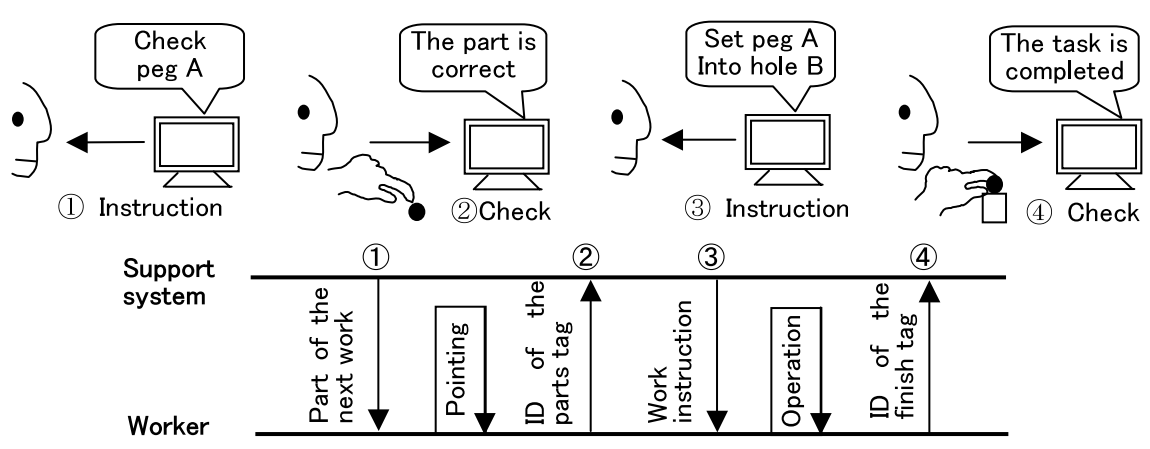

Fig. 1 Example of the interaction process between worker and support system 


\subsection{Using RFID to detect work information}

The RFID system consists of a RFID tag and $\mathrm{read} / \mathrm{write}$ device. The RFID tag has an antenna for receiving radio waves and a small IC chip for recording the ID number and some data. There are various shapes and sizes of RFID tags. A read/write device is used to access the tags without physical contact. The RFID system is widely used for baggage tags in logistics systems.

The possible usage of RFID to detect work information for checking assembly work is classified as follows.

\subsubsection{Detection of work piece Object identifica-} tion is a normal application of RFID. If the tag is set on a work piece and the ID number is recorded in a database that refers parts information, the system can retrieve data of the parts the worker is handling with.

\subsubsection{Detection of work point The distance at} which the read/write device can access the tag depends mainly on the power of the radio waves, and size and directivity of the antenna. If the position of a fixed tag and the possible read/write distance is known, the work point surrounding the detected tag can be estimated.

\subsubsection{Detection of assembly tool If a tag is set} on an assembly tool such as a screwdriver, the kind of tool being used by the worker can be determined when the tag is identified by the read/write device mounted on the worker's hand. If a numerous tags are set on the tool, there is a possibility of estimating the handling motion of the worker.

\subsubsection{Detection of work time If the worker al-} ways checks the tags just before treating the work piece, the time between two checks equals the work time. Moreover, if the ID number is recorded along with the time, the worker's name and other information, the record itself becomes an electronic work record.

\subsection{System configuration}

There are many types of RFID systems available. Each combination of a tag and a read/write device has different specifications in terms of capable read/write distance, read/write speed, recording capacity and other properties. Generally, a large antenna and powerful ra- dio waves result in a long read/write distance. However, metallic materials sometimes interfere with the capable read/write distance. Considering the wearable-device size and environmental issues related to mechanical products, a combination of tag and read/write device of $125 \mathrm{kHz}$ electromagnetic induction type, shown in Fig. 2, is chosen. The glass-type tag has a cylindrical shape. It has the advantage that it can be set in a narrow gap and on a steel part. The read/write device is a small modular one, and it communicates with a PC via an RS232 connection. Figure 2 (b) shows a photograph of the prototype system. The antenna is a coil type and is set on the worker's finger. ID number of tags can be read by touching the finger to a tag. The detectable distance between the tag and the antenna is about $10 \mathrm{~mm}$. Figure 3 shows the system configuration. The presentation device can be replaced with a headmounted display (HMD). The PC checks the detected ID number with a work sequence recorded in the database. If the expected ID number is detected, the next instruction is presented. The instruction is written in HTML form so it can be downloaded from a networked machine if needed. If an unexpected ID number is detected, an error message is presented.

\section{Measuring Human Property for Interaction De- sign}

In order to prepare a guide to set the interaction process, a simulation software similar to a computer game is used to measure human properties of memorization and learning. The test using software is useful for measuring human property in a constant situation. The software mea-

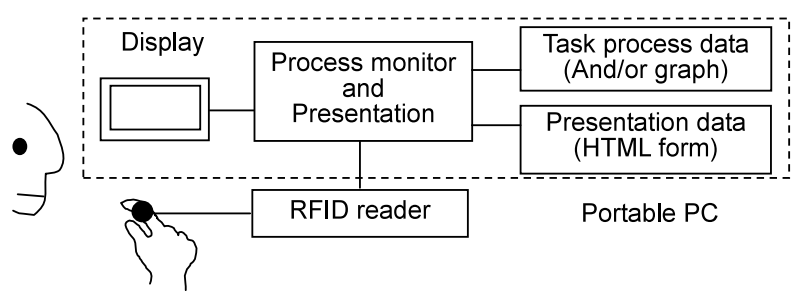

Fig. 3 System configuration of the prototype system

\section{RFID antenna set Display panel on worker's finger on portable PC}

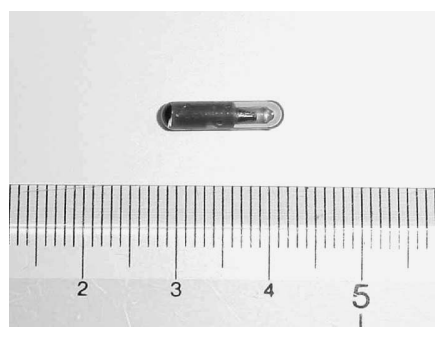

(a) RFID tag

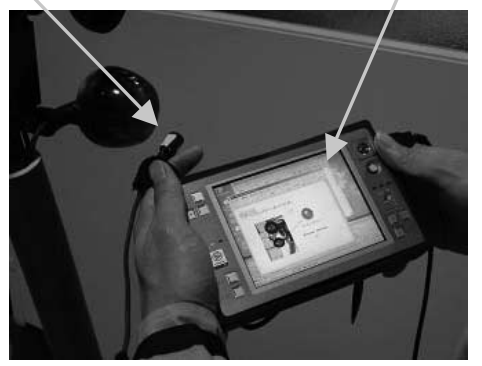

(b) Overview of the prototype system

Fig. 2 RFID system used in this prototype system 
sures the capacity for memorizing and recalling work sequences in a short time. The software generates an artificial work environment to measure the memorization ability of simple symbol orders. The memory capacity of a practical work sequence will be more than the result of this test because human memorizes practical work sequences often by connecting the sequence with the product structures. We can say that this test measures the minimum capacity of novice workers. There are two objectives in this experiment. One is to measure how long sequence the human can memorize and recall in a short time. Another is to evaluate the learning curve under the situation that the next task is given one by one. These experimental results will verify the learning effects in a work using the interactive support system.

\section{1 Work simulator}

In Fig. 4, the simulation window is shown. In this tester, a random sequence of 3 shapes: $O, \Delta, \square$, is prepared. The 3 shapes represent different tasks, and their order simulates a work sequence. The subject memorizes the sequence in 30 seconds. Then, the sequence is recalled, pushing the $\odot, \Delta$ or $\square$ button one by one within limited time for judgment. If a wrong button or no button is pushed, the answer is shown and the worker must push the button of next symbol. After pushing button, the worker must wait 3 to 5 seconds until the next task button becomes available. The waiting time simulates the work time. The limited time for judgment and the waiting time are variable according to the symbols, as shown in Table 2. These parameters are decided after some trial testing. Figure 5 shows the flowchart of this process. The number of symbol series is three in the first run. If a subject chooses all symbols correctly, a longer symbol series is attempted. Figure 4 (b) shows the modified software used in the learning test. The software has a checking function. When the checking button is pushed, the next symbol is displayed. This function simulates the work support system that presents the work sequence information.

\subsection{Experiment on memory capacity}

To begin with, an experiment is carried out to acquire basic data on how much task order a worker can memorize at a time. In this experiment, the maximum length of the work sequence that the worker can memorize and recall is measured. A subject is tested with not only one test window but also several test windows at a time to prevent the subject from memorizing particular symbol series. For some test windows, the recognition time and waiting time are multiplied with the number of windows. The result is evaluated using the following equation.

Table 2 Work time and time limit for work

\begin{tabular}{|c|c|c|}
\hline Symbol & Waiting time & Time limit \\
\hline$\bigcirc$ & 3 & 3 \\
\hline$\triangle$ & 4 & 4 \\
\hline$\square$ & 5 & 5 \\
\hline
\end{tabular}

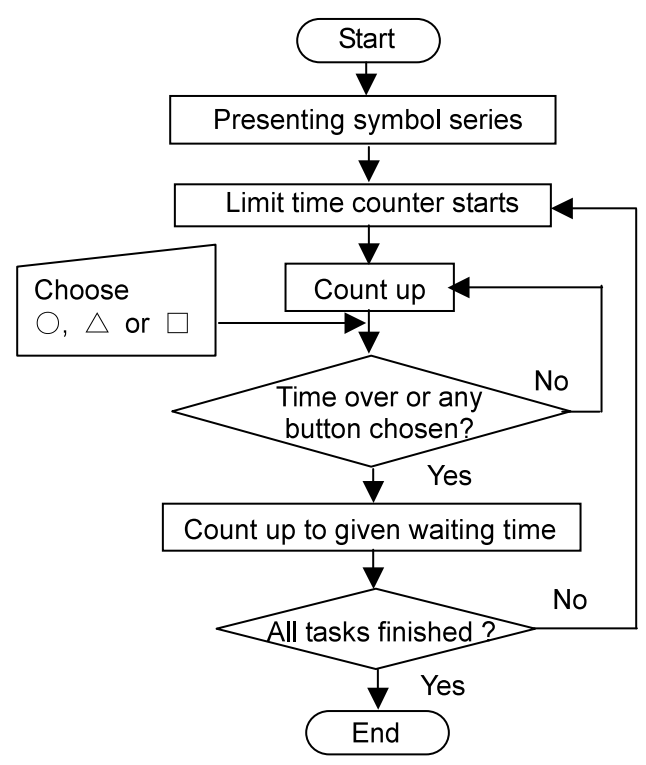

Fig. 5 Flowchart of the testing software

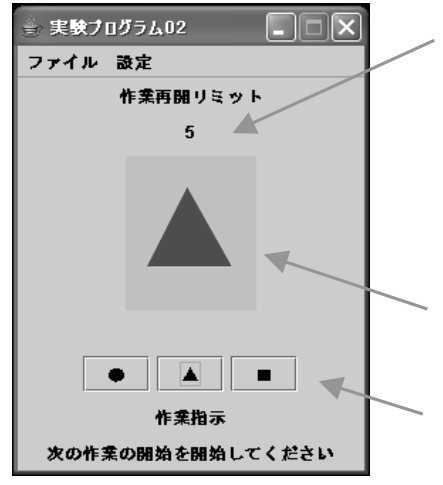

(a) Test window

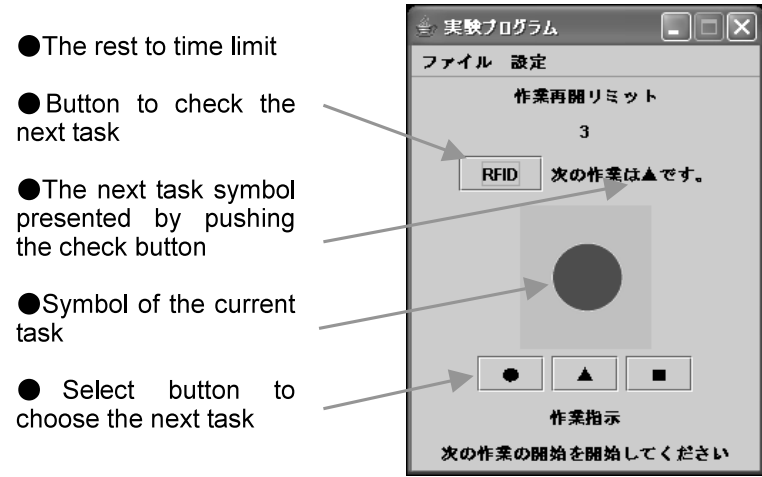

(b) Learning window

Fig. 4 Image of the simulation software to measure human property 
Memory capacity

$=$ Number of symbol series $\times$ Number of test windows

Memory capacity means the total length of symbol series that the human can memorize using immediate memory (working memory). The capacity of immediate memory is known to be the magical number seven plus or minus two $(7 \pm 2)^{(8)}$. However, the condition for memorizing a series of tasks is slightly different. Therefore, an experiment is conducted to measure the capacity in a similar situation to assembly work.

Twenty subjects who were all students of the university were tested with the number of windows from 1 to 4. Figure 6 shows the experimental results. We can see that the total capacity of memory, as expressed by Eq. (1) is approximately constant without regard to the number of task windows. The approximation curve is calculated with an inversely proportional function. This curve is drawn on Fig. 6. Since this curve is within standard deviations for all task window numbers, we can see that 11 is the average of the maximum capacity of memory in this test.

\subsection{Experiment on the work learning effect}

This experiment is conducted to evaluate the learning effect in work with the work instruction and checking process. The process seems to enhance the learning effect because more instruction information is given than that in the usual process. However, much instruction may cause workers to become dependent on given information and the decrease the activity for learning. Therefore, the learning effect in work with the instruction system should be evaluated by using the software. The checking button is added to the window, as shown in Fig. 4 (b). We call it the learning window, in contrast to the test window. Subjects must check the next task by pushing this button just before choosing the task button. The subject can choose correct button by choosing the displayed symbol. To evaluate the learning effects with respect to the amount of experience, the maximum length of a symbol series is measured using the test window after repeating check-and-select operations of the symbol series using the learning window

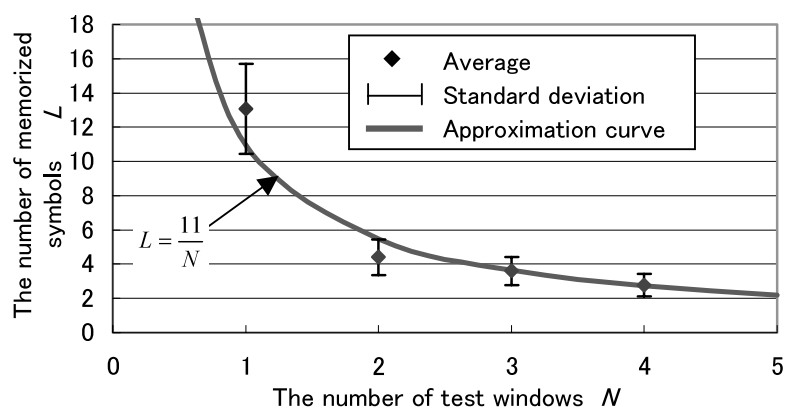

Fig. 6 Experimental results of memory capacity and approximation curve several times. Figure 7 shows the relationship between the number of repetitions and the maximum memory length of symbol series. The learning curve is approximated using a logarithm function. The result shows that repeating work increases the memory. In this case, the result of twice work is more than 11 , which is the result of 30 seconds of learning in advance. We can see that, for the worker, the work experience has the effect of reinforcing the memory of the work sequence. Therefore, the work support system is effective not only for reducing human error but also for learning the work process.

\section{Evaluation of the Interactive Work Support Sys- tem}

\subsection{Work object and interaction design}

As a case study of the interaction design and practical test of the work support system, the worker works with the prototype system described in section 3 . This system is applied to the assembly of the electric car as the work object of this experiment. This work consists of 8 tasks, and a total of 23 kinds of parts are used. Table 3 shows the list of assembly tasks, parts and work points identified by RFID.

In the interaction process, work instruction including the touching point is presented first. Second, the worker performs the indicated task and then returns a check signal by touching the indicated point. If the wrong tags are detected, an alarm message appears. If the correct tags are detected, the instruction for the next task is presented. This process is repeated until all tasks are finished. The checking period is an important factor for work efficiency. For example, checking every part such as a bolt and a nut consumes too much time. Therefore, it is significant that the checking process should be set according to the memory capacity of the work, and the frequency of the interaction is reduced according to the learning effect. We propose to set interaction processes so as to satisfy the following two rules.

(1) One work instruction includes no more than 11 new items.

(2) Interaction processes should be set until the total

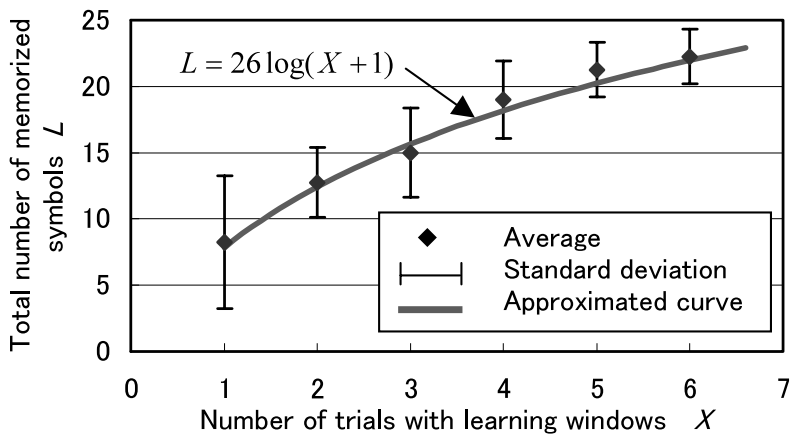

Fig. 7 Experimental result of learning and approximated curve 
Table 3 List of tasks, parts and RFID used in the experiment

\begin{tabular}{|c|c|c|c|c|}
\hline No & Task name & Parts & Check & RFID \\
\hline \multirow{4}{*}{1} & \multirow{4}{*}{$\begin{array}{l}\text { Wiper driving } \\
\text { device }\end{array}$} & \multirow{2}{*}{ Motor } & part & 1 \\
\hline & & & work point & 2 \\
\hline & & $(\mathrm{B} 01 \mathrm{~W} 01) \times 2$ & \begin{tabular}{|l|} 
part \\
\end{tabular} & 3,4 \\
\hline & & $(D) 1,0 v 01)^{n}$ & work point & 5,6 \\
\hline \multirow{2}{*}{2} & \multirow{2}{*}{ Wiper holder } & N01,G01,G02, & part & $7 \sim 10$ \\
\hline & & & work point & 11 \\
\hline \multirow{2}{*}{3} & \multirow{2}{*}{$\begin{array}{l}\text { Preparation of } \\
\text { roof assembly }\end{array}$} & \multirow{2}{*}{ W03×2 } & part & 12 \\
\hline & & & work point & 13,14 \\
\hline \multirow{4}{*}{4} & \multirow{4}{*}{ Roof assembly } & \multirow{2}{*}{ Roof } & part & 15 \\
\hline & & & work point & 16 \\
\hline & & \multirow{2}{*}{ B03,W04,W05 } & part & $17 \sim 19$ \\
\hline & & & work point & 20,21 \\
\hline \multirow{2}{*}{5} & \multirow{2}{*}{ Headlight } & \multirow{2}{*}{$\begin{array}{c}\text { Cover } \times 2, \\
(B 04, \text { W06 }) \times 2 \\
\end{array}$} & part & $22 \sim 24$ \\
\hline & & & work point & 25,26 \\
\hline \multirow{2}{*}{6} & \multirow{2}{*}{$\begin{array}{c}\text { Wiper } \\
\text { assembly }\end{array}$} & \multirow{2}{*}{ Wiper, B02 } & part & 27,28 \\
\hline & & & work point & 29 \\
\hline \multirow{4}{*}{7} & \multirow{4}{*}{ Break lamp } & \multirow{2}{*}{ Bulb A×2 } & \begin{tabular}{|c|} 
part \\
\end{tabular} & 30 \\
\hline & & & work point & 31,32 \\
\hline & & \multirow{2}{*}{$\begin{array}{c}\text { Cover } \times 2 \\
\text { B0 } 5 \times 4\end{array}$} & part & 33,34 \\
\hline & & & work point & 31,32 \\
\hline \multirow{4}{*}{8} & \multirow{4}{*}{ Winkers lamp } & \multirow{2}{*}{ Bulb $B \times 2$} & part & 35 \\
\hline & & & work point & 36,37 \\
\hline & & \multirow{2}{*}{$\begin{array}{c}\text { Cover } \times 2 \\
\text { B05 } \times 4\end{array}$} & \begin{tabular}{|l|} 
part \\
\end{tabular} & 38,39 \\
\hline & & & work point & 36,37 \\
\hline
\end{tabular}

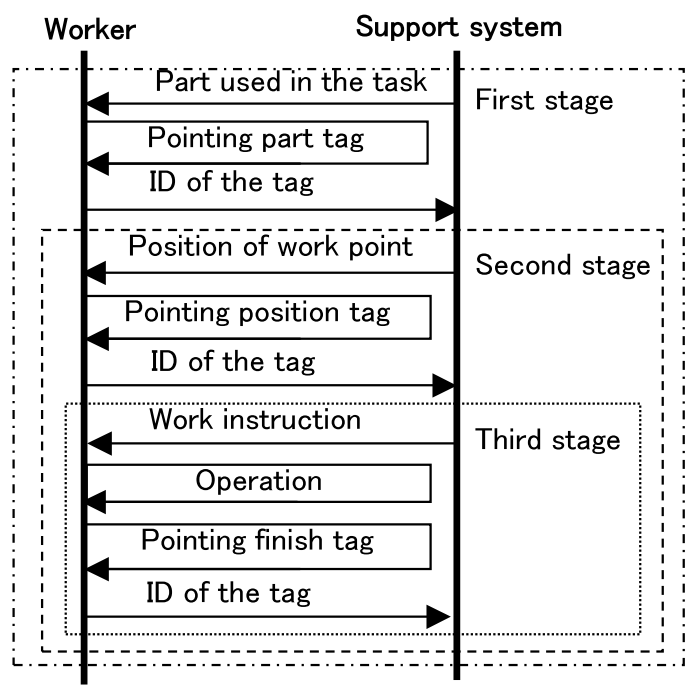

Fig. 8 Interaction process for each stage in the experiment

number of new items is below the learning curve.

where, item means a part or work point in a work sequence.

The total number of the kinds of parts is 23 . Because six parts, such as the roof, are clearly distinguishable, the worker must learn the remaining 17 kinds of parts in this work. Also, work points are easy to associate if the part is known. Considering these factors, it is thought that a worker must execute the work at least three times to learn all items while checking processes can be gradually reduced. Then, three stages of interaction patterns are prepared in this experiment. Figure 8 shows the interaction process of each stage. In the first stage, detailed work instruction is presented, and all parts and work points are

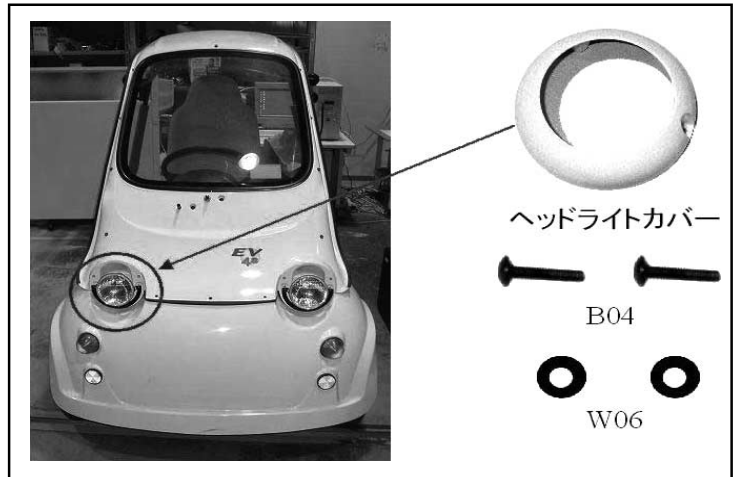

Fig. 9 Example of task instruction

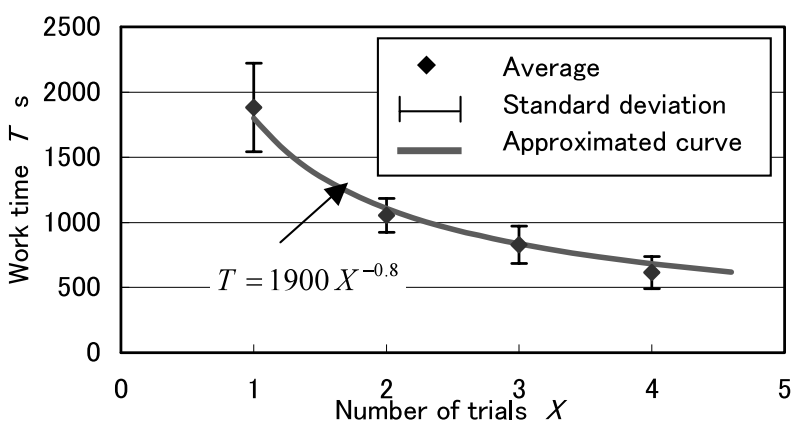

Fig. 10 Experimental result of work time over four trials

checked by RFID. In the second stage, detailed work instruction is presented and all parts are checked, but work position is not checked. In the third stage, a rough work process is presented and RFID is used only to input the task completion signal. Detailed instruction means presenting one instruction per kind of part. Rough instruction means presenting one instruction per task. Any rough instruction includes no more than 11 kinds of parts. Figure 9 shows an example of rough instruction. After workers execute the actual work with the support system three times, the fourth trial is performed without referring to any manual or receiving any support.

\subsection{Assembly experiment}

The experiment of actual assembly work is examined to confirm the effects of the support system. In this experiment, two things are confirmed. One is that the worker can complete the long sequence of actual tasks with the indication and checking process even if the worker is performing the task for the first time. The other thing is the learning effect of working with the work support system.

Four subjects executed the all tasks in Table 3 three times with interaction, mentioned in the section 5.1, and then executed the task again without the support system to test whether they learn the work. To evaluate the learning effects, the work time of each stage was measured.

All workers executed all tasks without any error. Figure 10 shows the average work time over four trials. The work time was gradually reduced at every stage. The re- 
sult depends on both learning effects and the reduction of checking time. From this result, the interaction design based on the capacity of human memory and the learning effects are validated. Moreover, the effectiveness of the work support system is confirmed.

\section{Conclusions}

(1) The work support system that contains the instruction and checking processes was proposed for novice workers to execute tasks without error even the first time.

( 2 ) The prototype system that uses RFID for the flexible interaction process design was realized, and checking processes using RFID were classified.

(3) The software that measures the capacity of human memory and learning in a simulated work process was developed. From experimental results of 20 subjects using this software, it was revealed that a total of 11 items of work can be memorized at one time. The learning effect of executing given tasks was observed and the learning curve was derived.

(4) The experiment on the work support system in an actual work process was conducted. The interaction process of the support system was designed in consideration of the human property revealed in the software test. As a result, all subjects were able to complete the tasks without error and learn them by the practical operations using the support system. From this result, the effectiveness of the work support system was confirmed.

\section{Acknowledgements}

The author would like to express his gratitude to
T.M.U. students Mr. Tatsuya Kaneko and Miss Keiko Shimizu for assistance with the experiments.

\section{References}

(1) McClellan, M., Applying Manufacturing Execution Systems, (1997), Saint Lucie Press.

( 2 ) Tateno, T., Misaki, D. and Aomura, S., Work Monitoring System of Human Motion with RFID, Proc. of JSME Int. Conf. on Leading Edge Manufacturing in 21st Century, (2003), pp.701-706.

(3) Raghavan, V., Molineros, J. and Sharma, R., Interactive Evaluation of Assembly Sequence Using Augmented Reality, IEEE Trans. on Robotics and Automation, Vol.15, No.3 (1999), pp.435-449.

(4) Carbonell, J.R., AI in CAI: An Artificial Intelligence Approach to Computer-Assisted Instruction, IEEE Trans. on Man-Machine Systems, Vol.MMS-11, No.4 (1970), pp.190-202.

( 5 ) Carroll, J.M., Learning by Doing with Simulated Intelligent Help, Communication of ACM, Vol.31, No.9 (1988), pp.1064-1079.

(6) Hayashi, M., Miyamoto, Y., Kataoka, M., Higuma, K. and Kuribayashi, E., Development of Training Simulator for Refuse Incineration Plants Using Virtual Reality, Proc. of JSME Int. Conf. of Power Engineering, (2003), pp.389-394.

(7) Newman, W.M. and Lamming, M.G., Interactive System Design, (1995), Addison-Wesley Pub.

( 8 ) Miller, G.A., The Magical Number Seven, Plus or Minus Two: Some limits on Our Capacity for Processing Information, The Psychological Review, Vol.63 (1956), pp.81-97. 